\title{
Halpern's iteration for Bregman strongly nonexpansive multi-valued mappings in reflexive Banach spaces with application
}

\section{Yi Li, Hongbo Liu and Kelong Zheng}

"Correspondence: liyi@swust.edu.cn School of Science, Southwest University of Science and Technology, Mianyang, Sichuan 621010, P.R. China

\begin{abstract}
Bregman strongly nonexpansive multi-valued mapping in reflexive Banach spaces is established. Under suitable limit conditions, some strong convergence theorems for modifying Halpern's iterations are proved. As an application, we utilize the main results to solve equilibrium problems in the framework of reflexive Banach spaces. The main results presented in the paper improve and extend the corresponding results in the work by Suthep et al. (Comput. Math. Appl. 64:489-499, 2012).
\end{abstract}

MSC: 47J05; 47H09; 49J25

Keywords: Bregman strongly nonexpansive multi-valued mapping; Legendre functions; Bregman projection; fixed point; Halpern's iteration sequence

\section{Introduction}

Let $D$ be a nonempty and closed subset of a real Banach space $X$. Let $N(D)$ and $C B(D)$ denote the family of nonempty subsets and nonempty, closed and bounded subsets of $D$, respectively. The Hausdorff metric on $C B(D)$ is defined by

$$
H\left(A_{1}, A_{2}\right)=\max \left\{\sup _{x \in A_{1}} d\left(x, A_{2}\right), \sup _{y \in A_{2}} d\left(y, A_{1}\right)\right\}
$$

for all $A_{1}, A_{2} \in C B(D)$, where $d\left(x, A_{1}\right)=\inf \left\{\|x-y\|, y \in A_{1}\right\}$. The multi-valued mapping $T: D \rightarrow C B(D)$ is called nonexpansive if $H(T x, T y) \leq\|x-y\|$ for all $x, y \in D$. An element $p \in D$ is called a fixed point of $T: D \rightarrow N(D)$ if $p \in T(p)$. The set of fixed points of $T$ is denoted by $F(T)$.

In recent years, several types of iterative schemes have been constructed and proposed in order to get strong convergence results for finding fixed points of nonexpansive mappings in various settings. One classical and effective iteration process is defined by

$$
x_{n+1}=\alpha_{n} u+\left(1-\alpha_{n}\right) T x_{n}, \quad x_{1}, u \in D,
$$

where $\alpha_{n} \in(0,1)$. Such a method was introduced in 1967 by Halpern [1] and is often called Halpern's iteration. In fact, he proved, in a real Hilbert space, strong convergence of $\left\{x_{n}\right\}$ to a fixed point of the nonexpansive mapping $T$, where $\alpha_{n}=n^{-a}, a \in(0,1)$.

Now, because of a simple construction, Halpern's iteration is widely used to approximate fixed points of nonexpansive mappings and other classes of nonlinear mappings. 
Reich [2] also extended the result of Halpern from Hilbert spaces to uniformly smooth Banach spaces. In 2012, Halpern's iteration for Bregman strongly nonexpansive mappings in reflexive Banach spaces was introduced and a strong convergence theorem for Bregman strongly nonexpansive mappings by Halpern's iteration in the framework of reflexive Banach spaces was proved.

In this paper, Bregman strongly nonexpansive multi-valued mappings in reflexive Banach spaces are introduced. Under suitable limit conditions, strong convergence theorems for the proposed modified Halpern's iterations are proved. As an application, we use our results to solve equilibrium problems in the framework of reflexive Banach spaces. The results presented in the paper improve and extend the corresponding results in [3].

\section{Preliminaries}

In the sequel, we begin by recalling some preliminaries and lemmas which will be used in our proofs. Let $X$ be a real reflexive Banach space with a norm $\|\cdot\|$ and let $X^{*}$ be the dual space of $X$. Let $f: X \rightarrow(-\infty,+\infty]$ be a proper, lower semi-continuous and convex function. We denote by $\operatorname{dom} f$ the domain of $f$.

Let $x \in \operatorname{intdom} f$. The subdifferential of $f$ at $x$ is the convex set defined by

$$
\partial f(x)=\left\{x^{*} \in X^{*}: f(x)+\left\langle x^{*}, y-x\right\rangle \leq f(y), \forall y \in X\right\} .
$$

The Fenchel conjugate of $f$ is the function $f^{*}: X^{*} \rightarrow(-\infty,+\infty]$ defined by

$$
f^{*}\left(x^{*}\right)=\sup \left\{\left\langle x^{*}, x\right\rangle-f(x): x \in X\right\} .
$$

We know that the Young-Fenchel inequality holds, that is,

$$
\left\langle x^{*}, x\right\rangle \leq f(x)+f^{*}\left(x^{*}\right), \quad \forall x \in X, x^{*} \in X^{*} .
$$

Furthermore, equality holds if $x^{*} \in \partial f(x)$ (see [4]). The set $\operatorname{lev}_{\leq}^{f}(r):=\{x \in X: f(x) \leq r\}$ for some $r \in R$ is called a sublevel of $f$.

A function $f$ on $X$ is called coercive [5] if the sublevel sets of $f$ are bounded, equivalently,

$$
\lim _{\|x\| \rightarrow+\infty} f(x)=+\infty
$$

A function $f$ on $X$ is said to be strongly coercive [6] if

$$
\lim _{\|x\| \rightarrow+\infty} \frac{f(x)}{\|x\|}=+\infty
$$

For any $x \in \operatorname{intdom} f$ and $y \in X$, the right-hand derivative of $f$ at $x$ in the direction $y$ is defined by

$$
f^{\circ}(x, y)=\lim _{t \rightarrow 0^{+}} \frac{f(x+t y)-f(x)}{t} .
$$

The function $f$ is said to be Gâteaux differentiable at $x$ if $\lim _{t \rightarrow 0^{+}} \frac{f(x+t y)-f(x)}{t}$ exists for any $y$. In this case, $f^{\circ}(x, y)$ coincides with $\nabla f(x)$, the value of the gradient $\nabla f(x)$ of $f$ at $x$. The 
function $f$ is said to be Gâteaux differentiable if it is Gâteaux differentiable for any $x \in$ $\operatorname{intdom} f$. The function $f$ is said to be Fréchet differentiable at $x$ if this limit is attained uniformly in $\|y\|=1$. Finally, $f$ is said to be uniformly Fréchet differentiable on a subset $D$ of $X$ if the limit is attained uniformly for $x \in D$ and $\|y\|=1$. It is known that if $f$ is Gâteaux differentiable (resp. Frêchet differentiable) on intdom $f$, then $f$ is continuous and its Gâteaux derivative $\nabla f$ is norm-to-weak* continuous (resp. continuous) on intdom $f$ (see [7] and [8]).

Definition 2.1 (cf. [9]) The function $f$ is said to be

(i) essentially smooth if $\partial f$ is both locally bounded and single-valued on its domain;

(ii) essentially strictly convex if $(\partial f)^{-1}$ is locally bounded on its domain and $\mathrm{f}$ is strictly convex on every convex subset of dom $\partial f$;

(iii) Legendre if it is both essentially smooth and essentially strictly convex.

Remark 2.1 ( $c f$. [10]) Let $X$ be a reflexive Banach space. Then we have

(a) $f$ is essentially smooth if and only if $f^{*}$ is essentially strictly convex;

(b) $(\partial f)^{-1}=\partial f^{*}$;

(c) $f$ is Legendre if and only if $f^{*}$ is Legendre;

(d) If $f$ is Legendre, then $\partial f$ is a bijection which satisfies

$$
\begin{aligned}
& \nabla f=\left(\nabla f^{*}\right)^{-1}, \quad \operatorname{ran} \nabla f=\operatorname{dom} \nabla f^{*}=\operatorname{intdom} f^{*} \text { and } \\
& \operatorname{ran} \nabla f^{*}=\operatorname{dom} \nabla f=\operatorname{intdom} f .
\end{aligned}
$$

Examples of Legendre functions can be found in [11]. One important and interesting Legendre function is $\frac{1}{p}\|\cdot\|^{p}(0<p<+\infty)$ when $X$ is a smooth and strictly convex Banach space. In this case, the gradient $\nabla f$ of $f$ is coincident with the generalized duality mapping of $X$, i.e., $\nabla f=J_{p}$. In particular, $\nabla f=I$ the identity mapping in Hilbert spaces. In this paper, we always assume that $f$ is Legendre.

The following crucial lemma was proved by Reich and Sabach [12].

Lemma 2.1 (cf. [12]) If $f: X \rightarrow R$ is uniformly Fréchet differentiable and bounded on bounded subsets of $X$, then $\nabla f$ is uniformly continuous on bounded subsets of $X$ from the strong topology of $X$ to the strong topology of $X^{*}$.

Let $f: X \rightarrow(-\infty,+\infty]$ be a convex and Gâteaux differentiable function. The function $D_{f}: \operatorname{dom} f \times \operatorname{intdom} f \rightarrow[0,+\infty)$, defined by

$$
D_{f}(y, x):=f(y)-f(x)-\langle\nabla f(x), y-x\rangle
$$

is called the Bregman distance with respect to $f$.

Recall that the Bregman projection [13] of $x \in \operatorname{intdom} f$ onto a nonempty, closed and convex set $D \subset \operatorname{dom} f$ is the necessarily unique vector $\operatorname{proj}_{D}^{f}(x) \in D$ (for convenience, here we use $P_{D}^{f}(x)$ for $\left.\operatorname{proj}_{D}^{f}(x)\right)$ satisfying

$$
D_{f}\left(\operatorname{proj}_{D}^{f}(x), x\right)=\inf \left\{D_{f}(y, x): y \in D\right\} .
$$


The modulus of total convexity of $f$ at $x \in \operatorname{intdom} f$ is the function $v_{f}(x, t):[0,+\infty) \rightarrow$ $[0,+\infty)$ defined by

$$
v_{f}(x, t):=\inf \left\{D_{f}(y, x): y \in \operatorname{dom} f,\|y-x\|=t\right\} .
$$

The function $f$ is called totally convex at $x$ if $v_{f}(x, t)>0$ whenever $t>0$. The function $f$ is called totally convex if it is totally convex at any point $x \in \operatorname{intdom} f$, and it is said to be totally convex on bounded sets if $v_{f}(B, t)>0$ for any nonempty bounded subset $B$ and $t>0$, where the modulus of total convexity of the function $f$ on the set $B$ is the function $v_{f}: \operatorname{intdom} f \times[0,+\infty) \rightarrow[0,+\infty)$ defined by

$$
v_{f}(B, t)=\inf \left\{v_{f}(x, t): x \in B \cap \operatorname{dom} f\right\} .
$$

We know that $f$ is totally convex on bounded sets if and only if $f$ is uniformly convex on bounded sets (see [14]).

Recall that the function $f$ is said to be sequentially consistent [14] if for any two sequences $\left\{x_{n}\right\}$ and $\left\{y_{n}\right\}$ in $X$, such that the first sequence is bounded, the following implication holds:

$$
\lim _{n \rightarrow+\infty} D_{f}\left(x_{n}, y_{n}\right)=0 \quad \Rightarrow \quad \lim _{n \rightarrow+\infty}\left\|x_{n}-y_{n}\right\|=0
$$

The following crucial lemma was proved by Butnariu and Iusem [15].

Lemma 2.2 (cf. [15]) The function $f$ is totally convex on bounded sets if and only if it is sequentially consistent.

Definition 2.2 (cf. [16]) Let $D$ be a convex subset of intdom $f$ and let $T$ be a multi-valued mapping of $D$. A point $p \in D$ is called an asymptotic fixed point of $T$ if $D$ contains a sequence $\left\{x_{n}\right\}$, which converges weakly to $p$, such that $d\left(x_{n}, T x_{n}\right) \rightarrow 0($ as $n \rightarrow \infty)$.

We denote by $\hat{F}(T)$ the set of asymptotic fixed points of $T$.

Definition 2.3 A multi-valued mapping $T: D \rightarrow N(D)$ with a nonempty fixed point set is said to be:

(i) Bregman strongly nonexpansive with respect to a nonempty $\hat{F}(T)$ if

$$
D_{f}(p, z) \leq D_{f}(p, x), \quad \forall x \in D, p \in \hat{F}(T), z \in T(x)
$$

and if, whenever $\left\{x_{n}\right\} \subset D$ is bounded, $p \in \hat{F}(T)$ and $\lim _{n \rightarrow \infty}\left[D_{f}\left(p, x_{n}\right)-D_{f}\left(p, z_{n}\right)\right]=0$, then $\lim _{n \rightarrow \infty} D_{f}\left(x_{n}, z_{n}\right)=0$, where $z_{n} \in T x_{n}$.

(ii) Bregman firmly nonexpansive if

$$
\left\langle\nabla f\left(x^{*}\right)-\nabla f\left(y^{*}\right), x^{*}-y^{*}\right\rangle \leq\left\langle\nabla f(x)-\nabla f(y), x^{*}-y^{*}\right\rangle, \quad \forall x, y \in D, x^{*} \in T x, y^{*} \in T y .
$$

In particular, the existence and approximation of Bregman firmly nonexpansive singlevalued mappings was studied in [16]. It is also known that if $T$ is Bregman firmly nonexpansive and $f$ is the Legendre function which is bounded, uniformly Fréchet differentiable 
and totally convex on bounded subsets of $X$, then $F(T)=\hat{F}(T)$ and $F(T)$ is closed and convex (see [16]). It also follows that every Bregman firmly nonexpansive mapping is Bregman strongly nonexpansive with respect to $F(T)=\hat{F}(T)$. The class of single-valued Bregman strongly nonexpansive mappings was introduced first in [17]. For a wealth of results concerning this class of mappings, see [18-22] and the references therein.

Remark 2.2 Let $X$ be a uniformly smooth and uniformly convex Banach space, and let $D$ be a nonempty, closed and convex subset. An operator $T: C \rightarrow N(D)$ is called a strongly relatively nonexpansive multi-valued mapping on $X$ if $\hat{F}(T) \neq \Phi$ and

$$
\phi(p, z) \leq \phi(p, x), \quad p \in \hat{F}(T), z \in T x,
$$

and if, whenever $\left\{x_{n}\right\} \subset D$ is bounded, $p \in \hat{F}(T)$ and $\lim _{n \rightarrow \infty}\left[\varphi\left(p, x_{n}\right)-\varphi\left(p, z_{n}\right)\right]=0$, then $\lim _{n \rightarrow \infty} \varphi\left(x_{n}, z_{n}\right)=0$, where $z_{n} \in T x_{n}$ and $\phi(x, y)=\|x\|^{2}-2\langle x, J y\rangle+\|y\|^{2}$.

Let $D$ be a nonempty, closed and convex subset of $X$. Let $f: X \rightarrow R$ be a Gâteaux differentiable and totally convex function and $x \in X$. It is known from [14] that $z=P_{D}^{f}(x)$ if and only if

$$
\langle\nabla f(x)-\nabla f(z), y-z\rangle \leq 0, \quad \forall y \in D .
$$

We also know the following characterization:

$$
D_{f}\left(y, P_{D}^{f}(x)\right)+D_{f}\left(P_{D}^{f}(x), x\right) \leq D_{f}(y, x), \quad \forall x, y \in D .
$$

Let $f: X \rightarrow R$ be a convex, Legendre and Gâteaux differentiable function. Following [23] and [24], we make use of the function $V_{f}: X \times X^{*} \rightarrow[0,+\infty)$ associated with $f$, which is defined by

$$
V_{f}\left(x, x^{*}\right)=f(x)+f^{*}\left(x^{*}\right)-\left\langle x, x^{*}\right\rangle, \quad \forall x \in X, x^{*} \in X^{*} .
$$

Then $V_{f}$ is nonnegative and $V_{f}\left(x, x^{*}\right)=D_{f}\left(x, \nabla f^{*}\left(x^{*}\right)\right)$ for all $x \in X$ and $x^{*} \in X^{*}$. Moreover, by the subdifferential inequality (see [22], Proposition 1(iii), p.1047),

$$
V_{f}\left(x, x^{*}\right)+\left\langle y^{*}, \nabla f^{*}\left(x^{*}\right)-x\right\rangle \leq V_{f}\left(x, x^{*}+y^{*}\right), \quad \forall x \in X, x^{*}, y^{*} \in X^{*} .
$$

In addition, if $f: X \rightarrow(-\infty,+\infty]$ is a proper and lower semi-continuous function, then $f^{*}: X^{*} \rightarrow(-\infty,+\infty]$ is a proper, weak* lower semi-continuous and convex function (see [25]). Hence $V_{f}$ is convex in the second variable (see [22], Proposition 1(i), p.1047). Thus,

$$
\begin{gathered}
D_{f}\left(z, \nabla f^{*}(t \nabla f(x)+(1-t) \nabla f(y))\right) \leq t D_{f}(z, x)+(1-t) D_{f}(z, y), \\
\forall t \in(0,1), \forall x, y \in X .
\end{gathered}
$$

The properties of the Bregman projection and the relative projection operators were studied in [14] and [26].

The following lemmas give us some nice properties of sequences of real numbers which will be useful for the forthcoming analysis. 
Lemma 2.3 (cf. [27], Lemma 2.1, p.76) Let $\left\{\alpha_{n}\right\}$ be a sequence of real numbers such that there exists a nondecreasing subsequence $\alpha_{n_{i}}$ of $\alpha_{n}$, that is, $\alpha_{n_{i}} \leq \alpha_{n_{i}+1}$ for all $i \in N$. Then there exists a nondecreasing subsequence $\left\{m_{k}\right\} \subset N$ such that $m_{k} \rightarrow \infty$, and the following properties are satisfied for all sufficiently large numbers sequence $k \subset N$ :

$$
\alpha_{m_{k}} \leq \alpha_{m_{k}+1} \text { and } \quad \alpha_{k} \leq \alpha_{m_{k}+1} \text {. }
$$

In fact, $m_{k}=\max \left\{j \leq k: \alpha_{j} \leq \alpha_{j+1}\right\}$.

Lemma 2.4 (see [3], Lemma 2.5, p.493) Assume that $\left\{\alpha_{n}\right\}$ is a sequence of nonnegative real numbers such that

$$
\alpha_{n+1} \leq\left(1-\gamma_{n}\right) \alpha_{n}+\gamma_{n} \delta_{n}
$$

where $\left\{\gamma_{n}\right\}$ is a sequence in $(0,1)$ and $\left\{\delta_{n}\right\}$ is a sequence such that

(a) $\lim _{n \rightarrow \infty} \gamma_{n}=0, \sum_{n=1}^{\infty} \gamma_{n}=\infty$;

(b) $\lim \sup _{n \rightarrow \infty} \delta_{n} \leq 0$.

Then $\lim _{n \rightarrow \infty} \alpha_{n}=0$.

\section{Main results}

To prove our main result, we first prove the following two propositions.

Proposition 3.1 Let $D$ be a nonempty, closed and convex subset of a real reflexive Banach space $X$. Let $f: X \rightarrow R$ be a Gâteaux differentiable and totally convex function, and let $T: D \rightarrow N(D)$ be a multi-valued mapping such that $F(T)=\hat{F}(T)$ is nonempty, closed and convex. Suppose that $u \in D$ and $\left\{x_{n}\right\}$ is a bounded sequence in $D$ such that $\lim _{n \rightarrow \infty} d\left(x_{n}, T x_{n}\right)=0$. Then

$$
\limsup _{n \rightarrow \infty}\left\langle\nabla f(u)-\nabla f(p), x_{n}-p\right\rangle \leq 0, \quad p=P_{F(T)}^{f}(u) .
$$

Proof Since $X$ is reflexive and $\left\{x_{n}\right\}$ is bounded, there exists a subsequence $\left\{x_{n_{k}}\right\} \subset\left\{x_{n}\right\}$ such that $x_{n_{k}} \rightarrow v \in D$ as $k \rightarrow \infty$ and

$$
\limsup _{n \rightarrow \infty}\left\langle\nabla f(u)-\nabla f(p), x_{n}-p\right\rangle=\langle\nabla f(u)-\nabla f(p), v-p\rangle .
$$

On the other hand, since $\lim _{k \rightarrow \infty} d\left(x_{n_{k}}, T_{n_{k}}\right)=0$, then $v \in \hat{F}(T)=F(T)$. By the definition of Bregman projection, we have

$$
\limsup _{n \rightarrow \infty}\left\langle\nabla f(u)-\nabla f(p), x_{n}-p\right\rangle=\langle\nabla f(u)-\nabla f(p), v-p\rangle \leq 0
$$

The proof of Proposition 3.1 is now completed.

The proof of the following result in the case of single-valued Bregman firmly nonexpansive mappings was done in ([16], Lemma 15.5, p.305). In the multi-valued case, the proof is identical and therefore we will omit the exact details. The interested reader will consult [16]. 
Proposition 3.2 Let $f: X \rightarrow(-\infty,+\infty]$ be a Legendre function and let $D$ be a nonempty, closed and convex subset of intdom $f$. Let $T: D \rightarrow N(D)$ be a Bregman firmly nonexpansive multi-valued mapping with respect to $f$. Then $F(T)$ is closed and convex.

Theorem 3.1 Let $X$ be a real reflexive Banach space and let $f: X \rightarrow(-\infty,+\infty]$ be a strongly coercive Legendre function which is bounded, uniformly Fréchet differentiable and totally convex on bounded subsets of X. Let D be a nonempty, closed and convex subset of intdom $f$ and let $T: D \rightarrow N(D)$ be a Bregman strongly nonexpansive multi-valued mapping on $X$ such that $F(T)=\hat{F}(T) \neq \emptyset$. Suppose that $u \in X$ and define the sequence $\left\{x_{n}\right\}$ by

$$
x_{1} \in D, \quad x_{n+1}=\nabla f^{*}\left(\alpha_{n} \nabla f(u)+\left(1-\alpha_{n}\right) \nabla f\left(z_{n}\right)\right), \quad z_{n} \in T x_{n}, n \geq 1,
$$

where $\alpha_{n} \in(0,1)$ satisfying $\lim _{n \rightarrow \infty} \alpha_{n}=0$ and $\sum_{n=1}^{\infty} \alpha_{n}=\infty$. Then $\left\{x_{n}\right\}$ strongly converges to $P_{F(T)}^{f}(u)$.

Proof First, by Proposition 3.2, we know that $F(T)$ is closed and convex. Letting $p=$ $P_{F(T)}^{f}(u) \in F(T)=\hat{F}(T)$, we have

$$
\begin{aligned}
D_{f}\left(p, x_{n+1}\right) & \leq \alpha_{n} D_{f}(p, u)+\left(1-\alpha_{n}\right) D_{f}\left(p, z_{n}\right) \\
& \leq \alpha_{n} D_{f}(p, u)+\left(1-\alpha_{n}\right) D_{f}\left(p, x_{n}\right) \\
& \leq \max \left\{D_{f}(p, u), D_{f}\left(p, x_{n}\right)\right\} .
\end{aligned}
$$

By the induction, the sequence $D_{f}\left(p, x_{n}\right)$ is bounded.

Next, we show that the sequence $\left\{x_{n}\right\}$ is also bounded. We follow the proof as in [16]. Since $D_{f}\left(p, x_{n}\right)$ is bounded, there exists $L>0$ such that

$$
f(p)-\left\langle\nabla f\left(x_{n}\right), p\right\rangle+f^{*}\left(\nabla f\left(x_{n}\right)\right)=V_{f}\left(p, \nabla f\left(x_{n}\right)\right)=D_{f}\left(p, x_{n}\right) \leq L .
$$

Hence $\left\{\nabla f\left(x_{n}\right)\right\}$ is contained in the sublevel set $\operatorname{lev}_{\leq}^{\psi}(L-f(p))$, where $\psi=f^{*}-\langle\cdot, p\rangle$. Since $f$ is lower semicontinuous, $f^{*}$ is weak* lower semicontinuous. Hence the function $\psi$ is coercive (see [4]). This shows that $\left\{\nabla f\left(x_{n}\right)\right\}$ is bounded. Since $f$ is strongly coercive, $f^{*}$ is bounded on bounded sets (see [9]). Hence $\nabla f^{*}$ is also bounded on bounded subsets of $E^{*}$ (see [15]). Since $f$ is a Legendre function, it follows that $x_{n}=\nabla f^{*}\left(\nabla f\left(x_{n}\right)\right), n \in \mathbb{N}$, is bounded. Therefore $\left\{x_{n}\right\}$ is bounded. So are $\left\{z_{n}\right\}$ and $\left\{\nabla f\left(z_{n}\right)\right\}$.

We next show that if there exists a subsequence $\left\{x_{n_{k}}\right\} \subset\left\{x_{n}\right\}$ such that

$$
\lim _{k \rightarrow \infty}\left[D_{f}\left(p, x_{n_{k}+1}\right)-D_{f}\left(p, x_{n_{k}}\right)\right]=0,
$$

then

$$
\lim _{k \rightarrow \infty}\left[D_{f}\left(p, z_{n_{k}}\right)-D_{f}\left(p, x_{n_{k}}\right)\right]=0,
$$

where $z_{n_{k}} \in T x_{n_{k}}$. 
Since $\left\{\nabla f\left(z_{n}\right)\right\}$ is bounded, we have from (3.2)

$$
\lim _{k \rightarrow \infty}\left\|\nabla f\left(x_{n_{k}+1}\right)-\nabla f\left(z_{n_{k}}\right)\right\|=\lim _{k \rightarrow \infty} \alpha_{n_{k}}\left\|\nabla f(u)-\nabla f\left(z_{n_{k}}\right)\right\|=0 .
$$

Since $f$ is strongly coercive and uniformly convex on bounded subsets of $X, f^{*}$ is uniformly Fréchet differentiable on bounded subsets of $X^{*}$ (see [6]). Moreover, $f^{*}$ is bounded on bounded sets. Since $f$ is Legendre, by Lemma 2.1, we obtain

$$
\lim _{k \rightarrow \infty}\left\|x_{n_{k}+1}-z_{n_{k}}\right\|=\lim _{k \rightarrow \infty}\left\|\nabla f^{*}\left(\nabla f\left(x_{n_{k}+1}\right)\right)-\nabla f^{*}\left(\nabla f\left(z_{n_{k}}\right)\right)\right\|=0 .
$$

On the other hand, since $f$ is uniformly Fréchet differentiable on bounded subsets of $X$, then $f$ is uniformly continuous on bounded subsets of $X$ (see [28]). It follows that

$$
\lim _{k \rightarrow \infty}\left|f\left(x_{n_{k}+1}\right)-f\left(z_{n_{k}}\right)\right|=0 .
$$

Since the following equality holds:

$$
\begin{aligned}
D_{f}\left(p, z_{n_{k}}\right)-D_{f}\left(p, x_{n_{k}}\right)= & f(p)-f\left(z_{n_{k}}\right)-\left\langle\nabla f\left(z_{n_{k}}\right), p-z_{n_{k}}\right\rangle-D_{f}\left(p, x_{n_{k}}\right) \\
= & f(p)-f\left(x_{n_{k}+1}\right)+f\left(x_{n_{k}+1}\right)-f\left(z_{n_{k}}\right)-\left\langle\nabla f\left(x_{n_{k}+1}\right), p-x_{n_{k}+1}\right\rangle \\
& +\left\langle\nabla f\left(x_{n_{k}+1}\right), p-x_{n_{k}+1}\right\rangle-\left\langle\nabla f\left(z_{n_{k}}\right), p-z_{n_{k}}\right\rangle-D_{f}\left(p, x_{n_{k}}\right) \\
= & D_{f}\left(p, x_{n_{k}+1}\right)+\left(f\left(x_{n_{k}+1}\right)-f\left(z_{n_{k}}\right)\right)+\left\langle\nabla f\left(x_{n_{k}+1}\right), p-x_{n_{k}+1}\right\rangle \\
& -\left\langle\nabla f\left(z_{n_{k}}\right), p-z_{n_{k}}\right\rangle-D_{f}\left(p, x_{n_{k}}\right) \\
= & \left(D_{f}\left(p, x_{n_{k}+1}\right)-D_{f}\left(p, x_{n_{k}}\right)\right)+\left(f\left(x_{n_{k}+1}\right)-f\left(z_{n_{k}}\right)\right)+\left\langle\nabla f\left(x_{n_{k}+1}\right)\right. \\
& \left.-\nabla f\left(z_{n_{k}}\right), p-x_{n_{k}+1}\right\rangle-\left\langle\nabla f\left(z_{n_{k}}\right), x_{n_{k}+1}-z_{n_{k}}\right\rangle,
\end{aligned}
$$

it follows from (3.3), (3.4) and (3.5) that

$$
\lim _{k \rightarrow \infty}\left(D_{f}\left(p, z_{n_{k}}\right)-D_{f}\left(p, x_{n_{k}}\right)\right)=0 .
$$

The rest of the proof will be divided into two parts.

Case 1 . Suppose that $\left\{D_{f}\left(p, x_{n}\right)\right\}$ is eventually decreasing, i.e., there exists a sufficiently large $k>0$ such that $D_{f}\left(p, x_{n}\right)>D_{f}\left(p, x_{n+1}\right)$ for all $n>k$. In this case, $\lim _{n \rightarrow \infty} D_{f}\left(p, x_{n}\right)$ exists. In this situation, we have that $\lim _{n \rightarrow \infty} D_{f}\left(p, x_{n}\right)$ exists. This shows that $\lim _{n \rightarrow \infty}\left(D_{f}\left(p, x_{n}\right)-\right.$ $\left.D_{f}\left(p, x_{n+1}\right)\right)=0$ and hence $\lim _{n \rightarrow \infty}\left(D_{f}\left(p, z_{n}\right)-D_{f}\left(p, x_{n}\right)\right)=0$.

Since $T$ is a Bregman strongly nonexpansive multi-valued mapping, then

$$
\lim _{n \rightarrow \infty}\left(D_{f}\left(x_{n}, z_{n}\right)\right)=0 .
$$

Since $f$ is totally convex on bounded subsets of $E$, by Lemma 2.2, we have

$$
\lim _{n \rightarrow \infty}\left\|x_{n}-z_{n}\right\|=0
$$

By Proposition 3.1, we obtain

$$
\limsup _{n \rightarrow \infty}\left\langle\nabla f(u)-\nabla f(p), x_{n}-p\right\rangle \leq 0 .
$$


Finally, we show that $x_{n} \rightarrow p$ as $n \rightarrow \infty$. Indeed

$$
\begin{aligned}
D_{f}\left(p, x_{n+1}\right)= & V_{f}\left(p, \alpha_{n} \nabla f(u)+\left(1-\alpha_{n}\right) \nabla f\left(z_{n}\right)\right) \\
\leq & V_{f}\left(p, \alpha_{n} \nabla f(u)+\left(1-\alpha_{n}\right) \nabla f\left(z_{n}\right)-\alpha_{n}(\nabla f(u)-\nabla f(p))\right) \\
& +\left\langle\alpha_{n}(\nabla f(u)-\nabla f(p)), x_{n+1}-p\right\rangle \\
= & V_{f}\left(p, \alpha_{n} \nabla f(p)+\left(1-\alpha_{n}\right) \nabla f\left(z_{n}\right)\right)+\alpha_{n}\left\langle\nabla f(u)-\nabla f(p), x_{n+1}-p\right\rangle \\
\leq & \alpha_{n} V_{f}(p, \nabla f(p))+\left(1-\alpha_{n}\right) V_{f}\left(p, \nabla f\left(z_{n}\right)\right)+\alpha_{n}\left\langle\nabla f(u)-\nabla f(p), x_{n+1}-p\right\rangle \\
= & \left(1-\alpha_{n}\right) D_{f}\left(p, z_{n}\right)+\alpha_{n}\left\langle\nabla f(u)-\nabla f(p), x_{n+1}-p\right\rangle \\
\leq & \left(1-\alpha_{n}\right) D_{f}\left(p, x_{n}\right)+\alpha_{n}\left\langle\nabla f(u)-\nabla f(p), x_{n+1}-p\right\rangle .
\end{aligned}
$$

By Lemma 2.4, we conclude that $\lim _{n \rightarrow \infty} D_{f}\left(p, x_{n}\right)=0$. Therefore, by Lemma 2.2, since $f$ is totally convex on bounded subsets of $X$, we obtain that $x_{n} \rightarrow p$ as $n \rightarrow \infty$.

Case 2. If $\left\{D_{f}\left(p, x_{n}\right)\right\}$ is not eventually decreasing, there exists a subsequence $\left\{D_{f}\left(p, x_{n_{j}}\right)\right\} \subset\left\{D_{f}\left(p, x_{n}\right)\right\}$ such that $D_{f}\left(p, x_{n_{j}}\right)<D_{f}\left(p, x_{n_{j}+1}\right)$ for all $j \in N$. By Lemma 2.3, there exists a strictly increasing sequence $\left\{m_{k}\right\}$ of positive integers such that the following properties hold for all $k \in N$ :

$$
D_{f}\left(p, x_{m_{k}}\right) \leq D_{f}\left(p, x_{m_{k}+1}\right), \quad D_{f}\left(p, x_{k}\right) \leq D_{f}\left(p, x_{m_{k}+1}\right) .
$$

Since the inequality $D_{f}\left(p, z_{n}\right)-D_{f}\left(p, x_{n}\right) \leq 0$ holds by Definition 2.3 , hence, by Lemma 2.3, we have

$$
\begin{aligned}
0 & \leq \lim _{k \rightarrow \infty}\left(D_{f}\left(p, x_{m_{k}+1}\right)-D_{f}\left(p, x_{m_{k}}\right)\right) \\
& \leq \limsup _{n \rightarrow \infty}\left(D_{f}\left(p, x_{n+1}\right)-D_{f}\left(p, x_{n}\right)\right) \\
& \leq \limsup _{n \rightarrow \infty}\left(\alpha_{n} D_{f}(p, u)+\left(1-\alpha_{n}\right) D_{f}\left(p, z_{n}\right)-D_{f}\left(p, x_{n}\right)\right) \\
& =\limsup _{n \rightarrow \infty}\left(\alpha_{n}\left(D_{f}(p, u)-D_{f}\left(p, z_{n}\right)\right)+D_{f}\left(p, z_{n}\right)-D_{f}\left(p, x_{n}\right)\right) \\
& \leq \limsup _{n \rightarrow \infty} \alpha_{n}\left(D_{f}(p, u)-D_{f}\left(p, z_{n}\right)\right)=0 .
\end{aligned}
$$

This implies that

$$
\limsup _{k \rightarrow \infty}\left(D_{f}\left(p, x_{m_{k}+1}\right)-D_{f}\left(p, x_{m_{k}}\right)\right)=0 .
$$

Following the proof of Case 1, we have

$$
\limsup _{k \rightarrow \infty}\left\langle\nabla f(u)-\nabla f(p), x_{m_{k}}-p\right\rangle \leq 0,
$$

and

$$
D_{f}\left(p, x_{m_{k}+1}\right) \leq\left(1-\alpha_{m_{k}}\right) D_{f}\left(p, x_{m_{k}}\right)+\alpha_{m_{k}}\left\langle\nabla f(u)-\nabla f(p), x_{m_{k}+1}-p\right\rangle .
$$


This implies that

$$
\begin{aligned}
\alpha_{m_{k}} D_{f}\left(p, x_{m_{k}}\right) & \leq D_{f}\left(p, x_{m_{k}}\right)-D_{f}\left(p, x_{m_{k}+1}\right)+\alpha_{m_{k}}\left\langle\nabla f(u)-\nabla f(p), x_{m_{k}+1}-p\right\rangle \\
& \leq \alpha_{m_{k}}\left\langle\nabla f(u)-\nabla f(p), x_{m_{k}+1}-p\right\rangle .
\end{aligned}
$$

Hence

$$
\lim _{k \rightarrow \infty} D_{f}\left(p, x_{m_{k}}\right)=0
$$

Using this and (3.5) together, we conclude that

$$
\limsup _{k \rightarrow \infty} D_{f}\left(p, x_{k}\right) \leq \lim _{k \rightarrow \infty} D_{f}\left(p, x_{m_{k}+1}\right)=0 .
$$

The proof of Theorem 3.1 is now completed.

As a direct consequence of Theorem 3.1 and Remark 2.2, we obtain the convergence result concerning strongly relatively nonexpansive multi-valued mappings in a uniformly smooth and uniformly convex Banach space.

Corollary 3.1 Let $X$ be a uniformly smooth and uniformly convex Banach space. Let D be a nonempty, closed and convex subset on $X$ and let $T: D \rightarrow N(D)$ be a strongly relatively nonexpansive multi-valued mapping on $X$ such that $F(T)=\hat{F}(T) \neq \emptyset$. Suppose that $u \in D$ and define the sequence $\left\{x_{n}\right\}$ as follows: $x_{1} \in D$ and

$$
x_{n+1}=J^{-1}\left(\alpha_{n} J(u)+\left(1-\alpha_{n}\right)\right) z_{n}, \quad z_{n} \in T x_{n}, \forall n \geq 1,
$$

where $\alpha_{n} \in(0,1)$ satisfying $\alpha_{n} \rightarrow 0$ and $\sum_{n=1}^{\infty} \alpha_{n}=\infty$. Then $\left\{x_{n}\right\}$ converges strongly to $\Pi_{F(T)} u$, where $\Pi_{F(T)}$ is the generalized projection onto $F(T)$.

\section{Application}

In this section, we give an application of Theorem 3.1, which is the equilibrium problems in the framework of reflexive Banach spaces.

Let $X$ be a smooth, strictly convex and reflexive Banach space, let $D$ be a nonempty, closed and convex subset of $X$, and let $G: D \times D \rightarrow R$ be a bifunction satisfying the conditions:

(A1) $G(x, x)=0$ for all $x \in D$;

(A2) $G(x, y)+G(y, x) \leq 0$ for any $x, y \in D$;

(A3) for each $x, y, z \in D, \lim _{t \rightarrow 0} G(t z+(1-t) x, y) \leq G(x, y)$;

(A4) for each given $x \in D$, the function $y \longmapsto f(x, y)$ is convex and lower semicontinuous.

The so-called equilibrium problem for $G$ is to find a $x^{*} \in D$ such that $G\left(x^{*}, y\right) \geq 0$ for each $y \in D$. The set of its solutions is denoted by $E P(G)$.

The resolvent of a bifunction $G[18]$ is the operator $\operatorname{Res}_{G}^{f}: X \rightarrow 2^{D}$ defined by

$$
\operatorname{Res}_{G}^{f}(x)=\{z \in D, G(z, y)+\langle\nabla f(z)-\nabla f(x) z, y-z\rangle \geq 0, \forall y \in D\}, \quad \forall x \in X .
$$


If $f: X \rightarrow(-\infty,+\infty]$ is a strongly coercive and Gâteaux differentiable function, and $G$ satisfies conditions (A1)-(A4), then $\operatorname{dom}\left(\operatorname{Res}_{G}^{f}\right)=X$ (see [18]). We also know:

(1) $\operatorname{Res}_{G}^{f}$ is single-valued;

(2) $\operatorname{Res}_{G}^{f}$ is a Bregman firmly nonexpansive mapping;

(3) $F\left(\operatorname{Res}_{G}^{f}\right)=E P(G)$;

(4) $E P(G)$ is a closed and convex subset of $D$;

(5) for all $x \in X$ and for all $p \in F\left(\operatorname{Res}_{G}^{f}\right)$, we have

$$
D_{f}\left(p, \operatorname{Res}_{G}^{f}(x)\right)+D_{f}\left(\operatorname{Res}_{G}^{f}(x), x\right) \leq D_{f}(p, x) .
$$

In addition, by Reich and Sabach [16], if $f$ is uniformly Fréchet differentiable and bounded on bounded subsets of $X$, then we have that $F\left(\operatorname{Res}_{G}^{f}\right)=\hat{F}\left(\operatorname{Res}_{G}^{f}\right)=E P(G)$ is closed and convex. Hence, by replacing $T=\operatorname{Res}_{G}^{f}$ in Theorem 3.1, we obtain the following result.

Theorem 4.1 Let D be a nonempty, closed and convex subset of a real reflexive Banach space X. Letf be a strongly coercive Legendre function which is bounded, uniformly Fréchet differentiable and totally convex on bounded subsets of X. Let $G: D \times D \rightarrow R$ be a bifunction which satisfies conditions (A1)-(A4) such that $E P(G) \neq \emptyset$. Suppose that $u \in X$ and define the sequence $\left\{x_{n}\right\}$ by

$$
x_{1} \in X, \quad x_{n+1}=\nabla f^{*}\left(\alpha_{n} \nabla f(u)+\left(1-\alpha_{n}\right) \nabla f\left(z_{n}\right), \quad z_{n} \in \operatorname{Res}_{G}^{f}\left(x_{n}\right), \forall n \geq 1,\right.
$$

where $\alpha_{n} \in(0,1)$ satisfying $\alpha_{n} \rightarrow 0$ and $\sum_{n=1}^{\infty} \alpha_{n}=\infty$. Then $\left\{x_{n}\right\}$ converges strongly to $P_{E P(G)}^{f} u$.

\section{Competing interests}

The authors declare that they have no competing interests.

\section{Authors' contributions}

All authors conceived of the study, participated in its design and coordination, drafted the manuscript, participated in the sequence alignment, and read and approved the final manuscript.

\section{Acknowledgements}

The authors are very grateful to both reviewers for carefully reading this paper and their comments. This work is supported by the Doctoral Program Research Foundation of Southwest University of Science and Technology (No. 11zx7129) and Applied Basic Research Project of Sichuan Province (No. 2013JY0096)

Received: 20 December 2012 Accepted: 5 July 2013 Published: 22 July 2013

\section{References}

1. Halpern, B: Fixed points of nonexpanding maps. Bull. Am. Math. Soc. 73, 957-961 (1967)

2. Reich, S: Approximating fixed points of nonexpansive mappings. Panam. Math. J. 4, 23-28 (1994)

3. Suthep, S, Yeol, JC, Prasit, C: Halpern's iteration for Bregman strongly nonexpansive mappings in reflexive Banach spaces. Comput. Math. Appl. 64, 489-499 (2012)

4. Rockafellar, RT: Level sets and continuity of conjugate convex functions. Trans. Am. Math. Soc. 123, 46-63 (1966)

5. Hiriart-Urruty, JB, Lemaréchal C: Convex Analysis and Minimization Algorithms II: Advanced Theory and Bundle Methods. Grundlehren der mathematischen Wissenschaften, vol. 306. Springer, Berlin (1993)

6. Zǎlinescu, C: Convex Analysis in General Vector Spaces. World Scientific, River Edge (2002)

7. Asplund, E, Rockafellar, RT: Gradients of convex functions. Trans. Am. Math. Soc. 139, 443-467 (1969)

8. Bonnans, JF, Shapiro, A: Perturbation Analysis of Optimization Problems. Springer, New York (2000)

9. Bauschke, HH, Borwein, JM, Combettes, PL: Essential smoothness, essential strict convexity and Legendre functions in Banach spaces. Commun. Contemp. Math. 3, 615-647 (2001)

10. Bauschke, $\mathrm{HH}$, Borwein, JM, Combettes, PL: Bregman monotone optimization algorithms. SIAM J. Control Optim. 42 , 596-636 (2003)

11. Bauschke, $\mathrm{HH}$, Borwein, JM: Legendre functions and the method of random Bregman projections. J. Convex Anal. 4, 27-67 (1997) 
12. Reich, S, Sabach, S: A strong convergence theorem for a proximal-type algorithm in reflexive Banach spaces. J. Nonlinear Convex Anal. 10, 471-485 (2009)

13. Bregman, LM: The relaxation method for finding the common point of convex sets and its application to the solution of problems in convex programming. U.S.S.R. Comput. Math. Math. Phys. 7, 200-217 (1967)

14. Butnariu, D, Resmerita, E: Bregman distances, totally convex functions and a method for solving operator equations in Banach spaces. Abstr. Appl. Anal. 2006, Article ID 84919 (2006)

15. Butnariu, D, lusem, AN: Totally Convex Functions for Fixed Points Computation and Infinite Dimensional Optimization. Kluwer Academic, Dordrecht (2000)

16. Reich, S, Sabach, S: Existence and approximation of fixed points of Bregman firmly nonexpansive mappings in reflexive Banach spaces. In: Fixed-Point Algorithms for Inverse Problems in Science and Engineering. Springer, New York (2011)

17. Reich, S: A weak convergence theorem for the alternating method with Bregman distances. In: Theory and Applications of Nonlinear Operators of Accretive and Monotone Type. Dekker, New York (1996)

18. Reich, S, Sabach, S: Two strong convergence theorems for Bregman strongly nonexpansive operators in reflexive Banach spaces. Nonlinear Anal. 73, 122-135 (2010)

19. Borwein, JM, Reich, S, Sabach, S: A characterization of Bregman firmly nonexpansive operators using a new monotonicity concept. J. Nonlinear Convex Anal. 12, 161-184 (2011)

20. Martín-Márquez, V, Reich, S, Sabach, S: Right Bregman nonexpansive operators in Banach spaces. Nonlinear Anal. 75 , 5448-5465 (2012)

21. Martín-Márquez, V, Reich, S, Sabach, S: Bregman strongly nonexpansive operators in reflexive Banach spaces. J. Math. Anal. Appl. 400, 597-614 (2013)

22. Martín-Márquez, V, Reich, S, Sabach, S: Iterative methods for approximating fixed points of Bregman nonexpansive operators. Discrete Contin. Dyn. Syst. 6, 1043-1063 (2013)

23. Alber, YI: Metric and generalized projection operators in Banach spaces: properties and applications. In: Kartsatos, AG (ed.) Theory and Applications of Nonlinear Operator of Accretive and Monotone Type. Dekker, New York (1996)

24. Censor, Y, Lent, A: An iterative row-action method for interval convex programming. J. Optim. Theory Appl. 34 , 321-353 (1981)

25. Phelps, RP: Convex Functions, Monotone Operators, and Differentiability, 2nd edn. Lecture Notes in Mathematics. Springer, Berlin (1993)

26. Kohsaka, F, Takahashi, W: Proximal point algorithms with Bregman functions in Banach spaces. J. Nonlinear Convex Anal. 6, 505-523 (2005)

27. Maingé, PE: The viscosity approximation process for quasi-nonexpansive mappings in Hilbert spaces. Comput. Math. Appl. 59, 74-79 (2010)

28. Ambrosetti, A, Prodi, G: A Primer of Nonlinear Analysis. Cambridge University Press, Cambridge (1993)

doi:10.1186/1687-1812-2013-197

Cite this article as: Li et al.: Halpern's iteration for Bregman strongly nonexpansive multi-valued mappings in reflexive Banach spaces with application. Fixed Point Theory and Applications 2013 2013:197.

\section{Submit your manuscript to a SpringerOpen ${ }^{\ominus}$ journal and benefit from:}

- Convenient online submission

- Rigorous peer review

- Immediate publication on acceptance

- Open access: articles freely available online

- High visibility within the field

- Retaining the copyright to your article 